\title{
How Inadequate Specification, Buggy Implementation, and Deficient Platform-Support Hinder Security
}

\author{
Omar Chowdhury \\ Department of Computer Science \\ The University of Iowa \\ Iowa City, Iowa \\ omar-chowdhury@uiowa.edu
}

\begin{abstract}
Developing a secure system (or, protocol) in general boils down to having a correct and robust specification which developers faithfully implement with the available platform support. Vulnerabilities can thus crop up due to inadequate specification, buggy implementations, or the lack of appropriate security constructs in the platform. In this talk, I will present examples of insecurity due to inadequate specification, wrong implementations, and deficient platform support. I will particularly focus on how automated reasoning and formal verification techniques can greatly contribute towards detecting vulnerabilities.

In the first example, I will show how 4G LTE telecommunication protocol specification lacks security considerations which can be exploited by adversaries to have catastrophic impacts [2]. Next, I will present how incorrect X.509 certificate validation implementations in open-source SSL/TLS libraries leave users prone to impersonation attacks [1]. Finally, I will conclude my talk with a discussion of how lack of hardware support makes enforcing Digital Rights Management (DRM) policies infeasible for mobile devices.
\end{abstract}

\section{CCS CONCEPTS}

- Security and privacy $\rightarrow$ Logic and verification; Security protocols; Mobile and wireless security; Mobile platform security;

\section{KEYWORDS}

Implementation bugs; Inconsistent Specification; X.509 Public-key Infrastructure; 4G LTE; Digital Rights Management; Inadequate platform constructs

\section{ACM Reference Format:}

Omar Chowdhury. 2018. How Inadequate Specification, Buggy Implementation, and Deficient Platform-Support Hinder Security. In SACMAT'18: 23rd ACM Symposium on Access Control Models \& Technologies, June 1315, 2018, Indianapolis, IN, USA. ACM, New York, NY, USA, 1 page. https: //doi.org/10.1145/3205977.3206002

\section{BIOGRAPHY}

Dr. Omar Haider Chowdhury is an Assistant Professor of Computer Science at the University of Iowa. Dr. Chowdhury's research

Permission to make digital or hard copies of part or all of this work for personal or classroom use is granted without fee provided that copies are not made or distributed for profit or commercial advantage and that copies bear this notice and the full citation on the first page. Copyrights for third-party components of this work must be honored

For all other uses, contact the owner/author(s).

SACMAT'18, June 13-15, 2018, Indianapolis, IN, USA

(C) 2018 Copyright held by the owner/author(s)

ACM ISBN 978-1-4503-5666-4/18/06.

https://doi.org/10.1145/3205977.3206002 focuses on leveraging formal machinery and techniques to solve practically-relevant security and privacy problems of emerging systems and protocols. At Iowa, Dr. Chowdhury currently co-directs the Computational Logic Center (CLC) and is also an active member of the Informatics Initiative (UI3).

Before joining the University of Iowa, he held post-doctoral researcher positions at Carnegie Mellon University and Purdue University. He received his Ph.D. in Computer Science from the University of Texas at San Antonio. Dr. Chowdhury's research is currently supported by the National Science Foundation (NSF) and Defense Advanced Research Projects Agency (DARPA).

\section{ACKNOWLEDGEMENT}

The results reported in this keynote is partially supported by the National Science Foundation (NSF) grant CNS-1657124. Any opinions, findings, or recommendations conveyed during the keynote are those of the author, and do not necessarily reflect those of NSF.

\section{REFERENCES}

[1] S. Y. Chau, O. Chowdhury, E. Hoque, H. Ge, A. Kate, C. Nita-Rotaru, and N. Li. 2017. SymCerts: Practical Symbolic Execution for Exposing Noncompliance in X.509 Certificate Validation Implementations. In 2017 IEEE Symposium on Security and Privacy (SP). 503-520.

[2] Syed Rafiul Hussain, Omar Chowdhury, Shagufta Mehnaz, and Elisa Bertino. 2018. LTEInspector: A Systematic Approach for Adversarial Testing of 4G LTE. In 2018 Network and Distributed System Security Symposium (NDSS). https://doi.org/10. $14722 /$ ndss.2018.23319 